\title{
Holographic superconductor in a deformed four-dimensional STU model
}

\author{
B. Pourhassan ${ }^{\mathrm{a}}$, M. M. Bagheri-Mohagheghi ${ }^{\mathrm{b}}$ \\ School of Physics, Damghan University, Damghan 3671641167, Iran
}

Received: 19 July 2017 / Accepted: 29 October 2017 / Published online: 11 November 2017

(C) The Author(s) 2017. This article is an open access publication

\begin{abstract}
In this paper, we consider a deformed STU model in four dimensions including both electric and magnetic charges. Using the AdS/CFT correspondence, we study holographic superconductors and obtain transport properties like electrical and thermal conductivities. We obtain transport properties in terms of the magnetic charge of the black hole and interpret it as the magnetic monopole of dual field theory. We find that the presence of the magnetic charge is necessary to have maximum conductivities, and the existence of a magnetic monopole with a critical charge $(137 e)$ to reach the maximum superconductivity is important. Also, we show that the thermal conductivity increases with increasing of the magnetic charge. It may be concluded that the origin of superconductivity is the magnetic monopole.
\end{abstract}

\section{Contents}

1 Introduction . . . . . . . . . . . . . . 1

2 Holographic superconductor . . . . . . . . . 2

3 Non-homogeneous STU black hole in four dimensions 4

4 Thermodynamics ............ 6

5 Conductivities ............ . 7

5.1 Electrical conductivity ......... 8

5.2 Thermal conductivity . . . . . . . . . 9

6 Conclusion ................ 9

References ................. 11

\section{Introduction}

The AdS/CFT correspondence, which relates conformal field theory (CFT) in $d$ dimensions and string theory in $(d+1)$ dimensional anti-de Sitter (AdS) space, is a powerful mathematical tool to study strongly correlated systems [1-3]. The

\footnotetext{
a e-mail: b.pourhassan@du.ac.ir

be-mail: bmohagheghi@du.ac.ir
}

extra dimension in the AdS side can be understood as the energy scale of the CFT on the boundary. The AdS/CFT correspondence is already used to study quark-gluon plasma (QGP) $[4,5]$, and it is possible to study the nuclear force using the AdS/CFT correspondence [6]. An interesting application of AdS/CFT is in condensed matter physics known as AdS/CMT, which claims that there is a dual gravitational description of superconductivity $[7,8]$, where the properties of a $(2+1)$-dimensional superconductor is reproduced using a neutral black hole with a charged scalar and only the Maxwell sector. So, it can be generalized to the case with an external magnetic field [9-12]. In order to review some important aspects of the theory of holographic superconductors, see Refs. [13,14].

Superconductivity is a phenomenon that has a history of about 100 years. Superconductivity is a state of a material in which $\rho=0$ (disappearance of resistivity). It occurs only below a certain temperature $\left(T_{C}\right)$, below a certain current level $\left(J_{C}\right)$, and below a certain magnetic field $\left(H_{C}\right)$. Superconductivity was first discovered by Onnes in 1911 for mercury. Later, much research has been done, theoretically and scientifically. The goal of the scientists was to justify this phenomenon by condensed matter and many-body models. From these kinds of models, we have the Fritz and Heinz two fluid model (1935), and phenomenological theories were proposed to explain superconductivity by Ginzburg and Landau (1950), and Cooper's BCS (Bardeen-Cooper-Schrieffer) pair model [15]. But Cooper's theory cannot justify superconductivity at higher temperatures $[16,17]$. On the other side, scientific work in this field is done on the basis of increasing the superconductivity transition temperature. In these days, scientists have reached a transition temperature of about 140 $\mathrm{K}$. But reaching higher temperatures is a great challenge for researchers.

In 1975, Price and Shirk [18] investigated the detection of a moving magnetic monopole. They conducted an experiment, which, with using a balloon-borne stack of Cherenkov 
film, emulsion, and 33 Lexan sheets, detected a very heavy particle having passed through them. For this heavy particle as a magnetic monopole, an electrical charge of $Q=137 e$ with a mass of 200 proton masses was obtained. This experiment is in agreement with investigations of charged particles by Malkus [19] in 1951, for arbitrary magnetic moment, moving simultaneously in the field of the monopole and an external electric field. It is concluded that the monopole can be coupled to matter with energies comparable to, but not significantly greater than, the chemical bond, reservations being made in the case of hydrogen where the lowest energy state depends upon the mass of the monopole. However, in 1982, the first results from a superconductive detector for moving magnetic monopoles, by Blas Cabrera, were reported [20]. Considering a magnetic charge moving at velocity $v$ along the axis of a superconducting wire ring of certain radius, the velocity- and mass-independent search for moving magnetic monopoles is being performed by continuously monitoring the current in a $20-\mathrm{cm}$-area superconducting loop. A single candidate event, consistent with one Dirac unit of the magnetic charge, has been detected during five runs.

The present work is done on the basis of a computing and analytical gravitation method and discusses the relation between the magnetic monopole model and superconductivity. The heavy monopole initially was suggested by 't Hooft and Polyakov in the framework of SU(2) gauge theories [2123]. Our model tries to link superconductivity to the magnetic monopole charge carrier's mobility, which has a major effect on the thermal and superconductive properties.

As is well known, ordinary superconductors are well described by the microscopic theory of superconductivity known as BCS theory [15]. But unusual superconductors including the pairing mechanism are not well understood using BCS theory and one needs another theoretical model of a strongly coupled system like the AdS/CFT correspondence. In order to have a realistic application of holography to superconductivity it is important to show how to introduce a dynamical (electromagnetic) gauge field in holographic superconductors [24-27]. In that case unusual magnetic materials with strongly correlated effects have been studied with the holographic duality [28], while an antiferromagnetism [29] as well as a paramagnetism [30] quantum phase transition can be considered by using holographic principles. According to the AdS/CFT dictionary, a black hole and charged scalar field are the holographic dual of the temperature and condensate of a superconductor, respectively [7]. In order to reproduce the superconductor phase diagram, one can consider a black hole with scalar hair at low temperature. The STU black hole is an important model with both electric and magnetic charges [31]. The STU model is just for $N=2$ supergravity [32,33]. It generally involves eight charges (four electric and four magnetic). There is complete STU symmetry, so the four charges have three charges that are on the same footing, while the last one has different couplings. The STU model can be interpreted in string theory by embedding. There are different ways to do this but the simplest, in type IIA on $T^{2} \times T^{2} \times T^{2}$, interprets the eight charges as D0, D2 (three of these, one for each $T^{2}$ ), D4 (three of these as well) and D6. The special case of the STU model in five dimensions with three electric charges which admits a chemical potential for the $U(1)^{3}$ symmetry already was used to study QGP; this is called the STU/QCD correspondence [34-38]. Also for $D=5, N=2$ the STU model is considered as a dual picture of a superfluid [39].

Motivated by the evidence of a superconductive detector for moving magnetic monopoles [20], we would like to investigate the effect of magnetic charge on the conductivities. The special case of the STU model in five dimensions already considered to study transport properties of superconductor [40]. In that case, various kinds of STU models are important from the AdS/CFT point of view and statistical analysis [41], because it is an extension of Yang-Mills theory to the case of a chemical potential. Recently, a deformation of the $N=2, D=4$ STU model, characterized by a nonhomogeneous special Kähler manifold, was considered to solve BPS attractor equations and to construct static supersymmetric black holes with radial symmetry [31]. In that case the relevant physical properties of the resulting black hole solution are explained and one can see that it is a four charge STU model, having three electric charges and one magnetic charge. Therefore, we consider for $N=2, D=4$ the STU model as a dual picture of a superconductor and investigate the effect of the magnetic charge on the transport properties. In other words, we would like to claim that the magnetic charge of the black hole is corresponding to the magnetic monopole in gauge theories.

This paper is organized as follows. In the next section, we review holographic superconductor and recall the basic equations. Then, in Sect. 3 we consider a non-homogeneous STU black hole in four dimensions and present important properties of the model. In Sect. 4 we discuss briefly the thermodynamics of the model and calculate some useful quantities to study the conductivities. In Sect. 5 we study the electrical end thermal conductivities and discuss the effect of the magnetic charge on them. Finally, in Sect. 6 we give a conclusion and a summary of results together with an outlook to future work.

\section{Holographic superconductor}

It has been shown that a gravitational background may be considered as a holographically dual picture of a superconductor [7], and it is known as holographic superconductor [42]. This means that the properties of strongly coupled superconductors in three dimensions may be described by four-dimensional classical general relativity. 
Perturbation equation helps us to obtain the transport coefficients from the general Lagrangian of the form

$L \equiv \frac{\mathcal{L}}{\sqrt{-g}}=R-\frac{1}{4} G_{i j} F_{\mu \nu}^{i} F^{\mu \nu j}+\cdots$,

where $R$ stands for the Ricci scalar and the dots denote scalar field and Chern-Simons terms; $F_{\mu \nu}$ is the field-strength tensor. The induced metric $G_{i j}$ related to the background metric $g_{i j}$ with determinant $g$ will be introduced later. In Ref. [40], the electrical and thermal conductivities of R-charged black hole in four, five, and seven dimensions is calculated for the general $D$-dimensional space-time,

$\mathrm{d} s^{2}=g_{t t} \mathrm{~d} t^{2}+g_{r r} \mathrm{~d} r^{2}+g_{x x} \mathrm{~d} \Omega_{D-2}^{2}$,

where $\mathrm{d} \Omega^{2}$ is $(D-2)$-dimensional space. We will use the results of Ref. [40] to study the transport properties of superconductor via a non-homogeneous deformation of the Rcharged black hole (STU black hole) in four dimensions. Perturbation equation for the gauge field of the general case can be written as follows [40]:

$\frac{\mathrm{d}}{\mathrm{d} r}\left(N_{i} \frac{\mathrm{d}}{\mathrm{d} r} \phi_{i}(r)\right)-\omega^{2} N_{i} g_{r r} g^{t t} \phi_{i}(r)+\sum_{j=1}^{m} M_{i j} \phi_{j}(r)=0$,

where

$N_{i}=\sqrt{-g} G_{i i} g^{x x} g^{r r}$

and

$M_{i j}=F_{r t}^{i} \sqrt{-g} G_{i i} g^{x x} g^{r r} G_{j j} F_{r t}^{j}$,

with the condition $M_{i j}=M_{j i}$. We can see that coupling of scalar field to the gauge field exists in the last term of the Eq. (2.3). Also, the scalar field $\phi_{i}$ is related to the $x$ component of the gauge field via

$A_{x}^{i}=\frac{\mu^{i}}{2} \Phi_{i}(r) \mathrm{e}^{i(q z-\omega t)}$,

where

$\phi_{i}(r)=\mu^{i} \Phi_{i}(r)$,

with $\omega$ and $q$ representing the frequency and momentum in the $z$ direction, respectively, and $\mu^{i}$ denotes the chemical potential. Hence, the chemical potential (Maxwell charge) is coupled to the scalar field $\Phi_{i}(r)$. It should be noted that the last term of Eq. (2.3) comes from the metric perturbation part, which is interpreted as an interaction between different gauge fields, so it will vanish for the case of non-interacting fields. Also the second term of Eq. (2.3) vanishes for the case of the low frequency limit $\left(\omega^{2} \rightarrow 0\right)$.

The fact is that the Maxwell equations imply that the phase of the complex scalar field must be constant. Hence, without loss of generality one can consider the scalar field as a real variable.

The diagonal and off diagonal components of conductivity are given by the following expressions, respectively:

$\sigma_{i i}=\frac{1}{8 \pi G_{D}}\left[\sqrt{\frac{g_{r r}}{-g_{t t}}} \frac{\sum_{k=1}^{m} N_{k}(r) \phi_{k}(r, \omega) \phi_{k}(r,-\omega)}{\left(\phi_{i}\right)^{0}\left(\phi_{i}\right)^{0}}\right]_{r=r_{+}}$

and

$\sigma_{i j}=\frac{1}{16 \pi G_{D}}\left[\sqrt{\frac{g_{r r}}{-g_{t t}}} \frac{\sum_{k=1}^{m} N_{k}(r) \phi_{k}(r, \omega) \phi_{k}(r,-\omega)}{\left(\phi_{i}\right)^{0}\left(\phi_{j}\right)^{0}}\right]_{r=r_{+}}$,

where $r_{+}$is the horizon radius, and $G_{D}$ is the $D$-dimensional Newtonian constant. We will use the above relations to study the electrical conductivity of a superconductor dual of the deformed STU model in four dimensions. It should be noted that the general form of the conductivities in the absence of a chemical potential is obtained by Ref. [43], but there is a Chern-Simons term in the STU model and we have both electric and magnetic charges, and therefore a chemical potential exists. So, one can use the general expression given by Ref. [40].

On the other hand, the coefficient of thermal conductivity given by

$\kappa_{T}=\left(\frac{\epsilon+P}{T}\right)^{2} \frac{1}{\sum_{i, j=1}^{m} \rho_{i}\left(\frac{i T}{\omega} G_{i j}(\omega)\right)^{-1} \rho_{j}}$,

where $\epsilon$ and $P$ are the local energy density and pressure respectively. Also, the $\rho_{i}$ are the charge densities satisfying the following thermodynamics equations:

$\epsilon+P=T s+\sum_{i=1}^{m} \mu^{i} \rho_{i}$

and

$\mathrm{d} \epsilon=T \mathrm{~d} s+\sum_{i=1}^{m} \mu^{i} \mathrm{~d} \rho_{i}$,

where $s$ and $\mu^{i}$ are the entropy and the chemical potential, respectively.

It should be noted here that there are two order parameters, the first is the coherence length $(\zeta)$, which is the scale of the variation of the order parameter. It indeed corresponds to the radial coordinate $r$ and hence $\phi(r)$ will be a complex order parameter to realize a holographic p-wave model $[44,45]$. The second is the London penetration depth $(\lambda)$, which characterizes the magnetic field penetration to the superconductor. It may be corresponding to the magnetic charge $p^{0}$, which will be introduced in the next section. 


\section{Non-homogeneous STU black hole in four dimensions}

As is well known, the STU background is an extension of the AdS-Schwarzschild background (which is the holographic dual of ordinary Yang-Mills theory) to include the chemical potential due to the presence of the black hole charge [34]. Hence, we expect that the 4D deformed background of the STU model could be a holographic dual of a superconductor as well as an ordinary AdS-Schwarzschild black hole background [7]. The main reason why we choose this special background is the presence of the magnetic charge. We would like to study the effect of the magnetic charge on superconductivity. As the background considered here is a solution of $N=2, D=4$ gauged supergravity coupled to Abelian vector multiples, it can reflect some properties of strongly coupled superconductors in $2+1$-dimensional space-time.

A non-homogeneous deformation of the STU model of $N=2, D=4$ gauged supergravity has been studied by Ref. [31], and we review some important properties of the model which will be useful to study holographic superconductor. The general form of the metric is given by

$\mathrm{d} s^{2}=-U(r) \mathrm{d} t^{2}+\frac{\mathrm{d} r^{2}+\psi(r) \mathrm{d} \Omega_{2}^{2}}{U(r)}$,

where $\mathrm{d} \Omega_{2}^{2}$ is the two-dimensional metric of surface. The determinant of the above line element is

$\sqrt{-g}=\frac{\psi(r)}{U(r)} f_{k}$

with $k=0, \pm 1$, where $f_{1}=\sin \theta, f_{0}=\theta$ and $f_{-1}=\sinh \theta$, which correspond to closed, flat and open universes.

The bosonic Lagrangian is given by [31],

$L=R-g_{i j} \partial_{\mu} z^{i} \partial^{\mu} \bar{z}^{j}-\frac{1}{4} \mathcal{I}_{\Lambda \Sigma} F_{\mu \nu}^{\Lambda} F^{\mu \nu \Sigma}+\cdots$,

where

$\mathcal{I}_{\Lambda \Sigma}=\frac{1}{4} \mathrm{e}^{-\mathcal{K}}\left(\begin{array}{cc}1 & 0 \\ 0 & 4 g_{i j}\end{array}\right)$

with

$$
\begin{aligned}
g_{i j}= & \frac{1}{2\left(\lambda^{1} \lambda^{2} \lambda^{3}-\frac{A}{3}\left(\lambda^{3}\right)^{3}\right)^{2}} \\
& \times\left(\begin{array}{ccc}
\left(\lambda^{2}\right)^{2}\left(\lambda^{3}\right)^{2} & \frac{A}{3}\left(\lambda^{3}\right)^{4} & -\frac{2 A}{3} \lambda^{2}\left(\lambda^{3}\right)^{3} \\
\frac{A}{3}\left(\lambda^{3}\right)^{4} & \left(\lambda^{1}\right)^{2}\left(\lambda^{3}\right)^{2} & -\frac{2^{A}}{3} \lambda^{1}\left(\lambda^{3}\right)^{3} \\
-\frac{2 A}{3} \lambda^{2}\left(\lambda^{3}\right)^{3} & -\frac{2 A}{3} \lambda^{1}\left(\lambda^{3}\right)^{3} & \left(\lambda^{1}\right)^{2}\left(\lambda^{2}\right)^{2}+\frac{A^{2}}{3}\left(\lambda^{3}\right)^{4}
\end{array}\right)
\end{aligned}
$$

and

$\mathrm{e}^{-\mathcal{K}}=8\left(\lambda^{1} \lambda^{2} \lambda^{3}-\frac{A}{3}\left(\lambda^{3}\right)^{3}\right)$, where $A$ is deformation parameter which may be fixed as unit, so $A=0$ gives us the ordinary STU model. The superpotential is specified by the dyonic Fayet-Iliopoulos (FI) gauging with FI parameters $\left(g^{\Lambda}, g_{\Lambda}\right)$. Also, in this model both magnetic $\left(p^{\Lambda}\right)$ and electric $\left(q^{\Lambda}\right)$ charges are possible. We will consider only the non-zero charges $p^{0}, q_{1}, q_{2}$ and $q_{3}$, which means that we have one magnetic and three electric charges. We are interested in the case of a positive magnetic charge and consider it as our physical case, while for the electrical charge we are free to choose any positive or negative values. This choice requires $\left(g_{0} p^{0}-g^{i} q_{i}=-k\right.$, where $\left.k=0, \pm 1\right)$ that some FI parameters vanish, so we have only $g^{1}, g^{2}, g^{3}$ and $g_{0}$, which are interpreted as gauge coupling constants (all positive) and related to the scalar fields $\lambda^{1}, \lambda^{2}$ and $\lambda^{3}$, which we will address later. In that case, the full black hole solution of the non-homogeneous STU model is given by

$\psi(R)=\left(a r^{2}-c\right)^{2}$

and

$U(r)=\frac{2 g_{0} g^{3}\left(a r^{2}-c\right)^{2}}{\lambda_{\infty}^{3}\left(a r-g_{0} \beta^{0}-\frac{g_{0} \beta_{3}}{\left(\lambda_{\infty}^{3}\right)^{2}}\right) \sqrt{\left(a r+2 g_{0} \beta^{0}\right)\left(a r+\frac{2 g_{0} \beta_{3}}{\left(\lambda_{\infty}^{3}\right)^{2}}\right)}}$,

where $a$ (with the length dimension) and $c$ (with the inverse of length dimension) are positive constants and

$\lambda_{\infty}^{3}=\sqrt{\frac{g_{0} g^{3}}{g^{1} g^{2}-\frac{A}{3}\left(g^{3}\right)^{2}}}$,

is the asymptotic value of $\lambda^{3}$. Also, $\beta^{0}$ and $\beta_{i}$ with $i=1,2,3$, are constants [46]. It is found that

$\lambda^{1}=\frac{a \frac{g^{1}}{g^{3}}\left(\lambda_{\infty}^{3}\right)^{2} r-g_{0} \beta_{3}\left(\frac{g^{1}}{g^{3}}-A \frac{g^{3}}{g^{2}}\right)-\beta^{0} \frac{\left(g_{0}\right)^{2}}{g^{2}}}{\sqrt{\left(2 g_{0} \beta^{0}+a r\right)\left(2 g_{0} \beta_{3}+\operatorname{ar}\left(\lambda_{\infty}^{3}\right)^{2}\right)}}$,

$\lambda^{2}=\frac{g^{2}}{g^{3}} \lambda^{3}$

$\lambda^{3}=\lambda_{\infty}^{3} \sqrt{\frac{a r+\frac{2 g_{0} \beta_{3}}{\left(\lambda_{\infty}^{3}\right)^{2}}}{a r+2 g_{0} \beta^{0}}}$.

In that case all quantities are described by $\beta^{0}, \beta_{i}, a, c, p^{0}$ and $q_{i}$.

The black hole horizon is given by

$r_{+}=\sqrt{\frac{c}{a}}$.

Now, we can discuss the perturbation equation (2.3) and obtain $\phi_{i}$ with $i=0,1,2,3$ corresponding to one magnetic and three electric charges of black hole. 

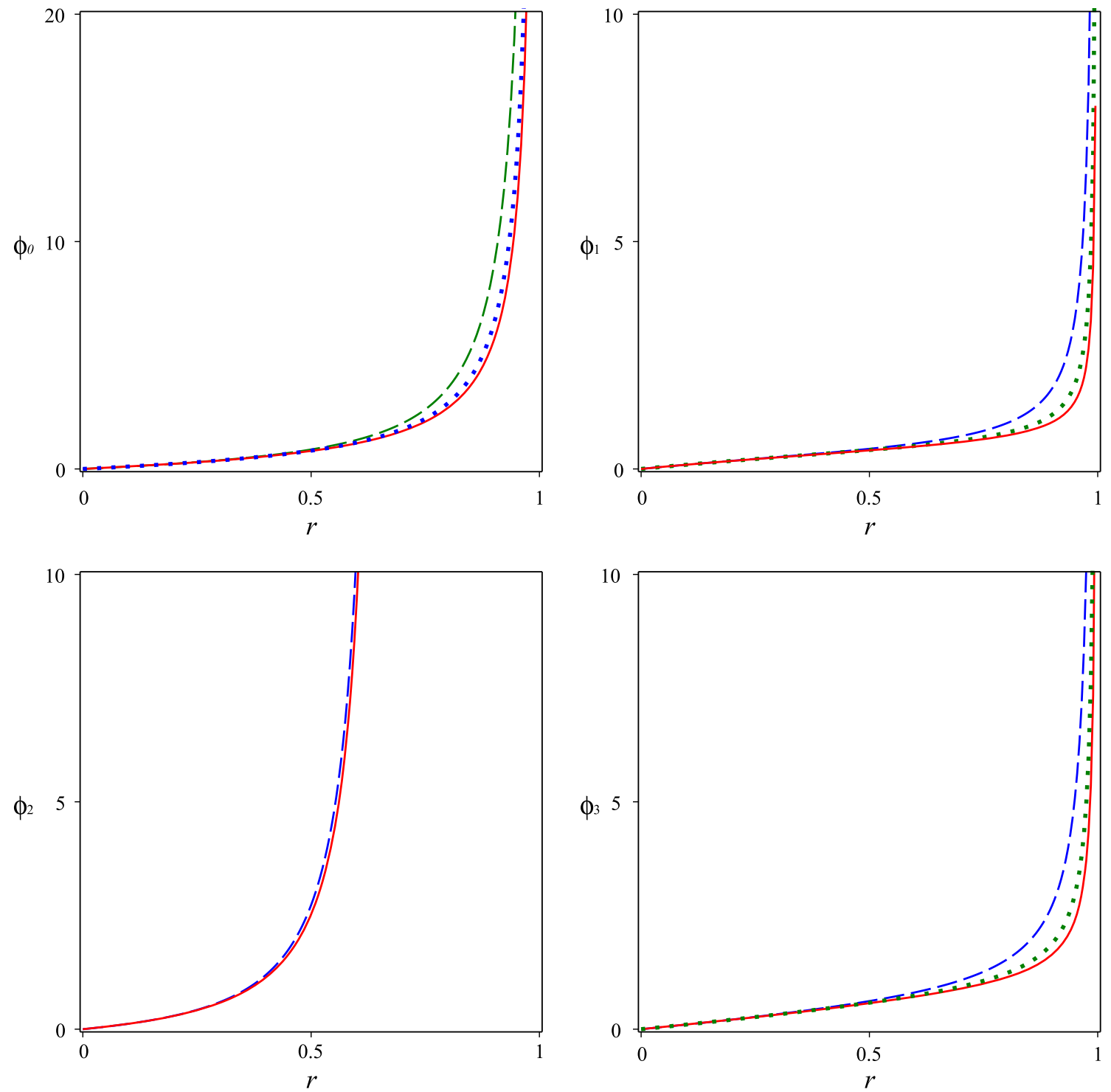

Fig. 1 Typical behavior of scalar fields in terms of $r\left(r \leq r_{+}\right)$with initial values $\phi(0)=0$ and $\phi^{\prime}(0)=1$. All model parameters are set to unity $\left(a=c=A=g_{0}=g^{1}=g^{2}=g^{3}=\beta^{0}=\beta_{3}=f_{k}=1\right) . \omega=1$ (dashed blue), $\omega=0.5$ (dotted green), $\omega=1$ (solid red)

Using Eq. (2.4) it is easy to find that

$$
\begin{aligned}
& N_{0}=2 f_{k}\left(\lambda^{1} \lambda^{2} \lambda^{3}-\frac{A}{3}\left(\lambda^{3}\right)^{3}\right) U(r), \\
& N_{1}=\frac{4 f_{k}\left(\lambda^{2}\right)^{2}\left(\lambda^{3}\right)^{2}}{\left(\lambda^{1} \lambda^{2} \lambda^{3}-\frac{A}{3}\left(\lambda^{3}\right)^{3}\right)} U(r), \\
& N_{2}=\frac{4 f_{k}\left(\lambda^{1}\right)^{2}\left(\lambda^{3}\right)^{2}}{\left(\lambda^{1} \lambda^{2} \lambda^{3}-\frac{A}{3}\left(\lambda^{3}\right)^{3}\right)} U(r),
\end{aligned}
$$

and

$$
N_{3}=\frac{4 f_{k}\left(\left(\lambda^{1}\right)^{2}\left(\lambda^{2}\right)^{2}+\frac{A^{2}}{3}\left(\lambda^{3}\right)^{4}\right)}{\left(\lambda^{1} \lambda^{2} \lambda^{3}-\frac{A}{3}\left(\lambda^{3}\right)^{3}\right)} U(r),
$$

where $U(r)$ is given by Eq. (3.8). Then the non-interacting version of Eq. (2.3) reads

$$
\frac{\mathrm{d}}{\mathrm{d} r}\left(N_{i} \frac{\mathrm{d}}{\mathrm{d} r} \phi_{i}(r)\right)+\omega^{2} N_{i} \frac{\phi_{i}(r)}{U(r)}=0 .
$$

So, we have four different equations to obtain $\phi_{0}(r), \phi_{1}(r)$, $\phi_{2}(r)$, and $\phi_{3}(r)$. In the simplest case of a low frequency we can neglect $\omega^{2}$. In that case one can obtain

$\phi_{i}(r)=C_{1} \int N_{i}^{-1} \mathrm{~d} r+C_{2}$,

where $C_{1}$ and $C_{2}$ are integration constants.

Numerically, we solve Eq. (3.16) and obtain the behavior of $\phi_{i}$ with respect to $r\left(r<r_{+}\right)$as illustrated by the plots of Fig. 1. We find that the presence of all FI parameters is 
necessary to obtain a finite and real value of the scalar fields. It is illustrated that scalar fields asymptotically yield infinity near the black hole horizon $\left(r_{+}=1\right.$ for $\left.a=c=1\right)$. We have shown that $\phi_{2}>\phi_{0}>\phi_{3}>\phi_{1}$. It is clear that the $\phi_{i}$ are increasing functions of $r$ inside the black hole. The behavior of the scalar fields is in agreement with the results of Ref. [40] in the region of $r<r_{+}$. We also find the effect of deformation on decreasing the value of the scalar field. It means that for bigger $A$ we have smaller $\phi_{i}$.

It is desirable that the scalar field should be well defined outside the horizon with positive value. In the low frequency limit, one can use Eq. (3.17) and obtain asymptotic values of the scalar fields for the large $r$ to find the behavior of the scalar field as illustrated by Fig. 2. We obtain plots corresponding to $A=1$, but the situation is similar for $A=-1$, which is used in Ref. [40]. We will show later that a negative value is suitable for the deformation parameter $A$ as used in Ref. [40]. So the boundary value of the scalar field $\left(\phi_{i}\right)^{0}$ is a constant (may be zero or one), for example $\left(\phi_{i}\right)^{0} \approx 1$ for the given value of model parameters as Figs. 1 and 2 .

In agreement with Ref. [40] and coincident with both large and small $r$ we can propose the following function for the scalar field:

$\phi_{i}(r)=\frac{a_{i}+b_{i} r}{c_{i}+d_{i} r}$

with $a_{i}<0, b_{i}<0, c_{i}<0$ and $d_{i}>0$. It indeed corresponds to $C_{2}=0$ of the solution (3.17). However, we can consider it as the general behavior of the scalar field satisfying the differential equation (3.16) for the non-interacting case with $\omega^{2} \rightarrow 0$.

\section{Thermodynamics}

In this section we will study thermodynamics properties of our system near the equilibrium. Near the horizon we have an infinitesimal temperature which is a decreasing function of $r$, and the black hole entropy is given by [40]

$s=\frac{\pi}{3} \frac{\mathcal{A}}{p^{0}}$

where the black hole horizon area is given by

$$
\begin{aligned}
\mathcal{A}= & 2\left(-p^{0} q_{3}\left[\left(-p^{0} q_{3}\right)^{2}+12\left(p^{0}\right)^{2} q_{1} q_{2}\right]\right. \\
& \left.+\left[\left(-p^{0} q_{3}\right)^{2}-4\left(p^{0}\right)^{2} q_{1} q_{2}\right]^{\frac{3}{2}}\right),
\end{aligned}
$$

with

$$
p^{0}=\frac{a c}{2 g_{0}}-2 g_{0}\left(\beta^{0}\right)^{2}
$$

as the magnetic charge and

$$
\begin{aligned}
q_{1}= & 2\left(\beta_{3}\right)^{2} \frac{g^{2}}{\left(g^{3}\right)^{2}}\left(g^{1} g^{2}-\frac{A}{3}\left(g^{3}\right)^{2}\right) \\
& -g^{2} \frac{a c}{2\left(g^{1} g^{2}-\frac{A}{3}\left(g^{3}\right)^{2}\right)}, \\
q_{2}= & \frac{1}{2 g^{2}}\left(\beta^{0} g_{0}+\beta_{3} \frac{g^{1} g^{2}}{g^{3}}\right)^{2}-g^{1} \frac{a c}{2\left(g^{1} g^{2}-\frac{A}{3}\left(g^{3}\right)\right.} \\
& +\frac{A}{3} \beta_{3} \frac{g^{3}}{g^{2}}\left(\beta_{3} \frac{g^{1} g^{2}}{g^{3}}-\beta^{0} g_{0}-\frac{A}{2} \beta_{3} g^{3}\right), \\
q_{3}= & \frac{g^{2}}{g^{3}} q_{2}-A \frac{g^{3}}{g^{2}} q_{1},
\end{aligned}
$$

as the electric charges.

In the case of positive magnetic charge ( $\left.p^{0} \geq 0\right)$, Eq. (4.3) requires that $g_{0} \leq \frac{a c}{4\left(\beta^{0}\right)^{2}}$. If we choose a unit value for the parameters (as selected in figures), then we should have $g_{0} \leq$ 0.5. So we use this as the allowed regions in the figures.

In Fig. 3 we show the values of the magnetic and the electric charges for the case of $a=c=\beta^{0}$ corresponding to $r_{+}=1$. According to Fig. $3 a$ it is clear that an infinitesimal $g_{0}$ yields a large positive value for the magnetic charge, while a large value of $g_{0}$ yields a large negative value for the magnetic charge, which is not our physical case (see Eq. (4.3)) and we only focus on the regions between $0 \leq g_{0} \leq 0.5$. It is clear that the magnetic charge vanishes for $g_{0}= \pm \frac{\sqrt{a c}}{2 \beta^{0}}$. As initial assumption the FI parameters all are positive, so a positive sign is acceptable and $g_{0}=\frac{\sqrt{a c}}{2 \beta^{0}}$ is corresponding to a zero magnetic charge. In the case of $a=c=\beta^{0}$ we have $g_{0}=0.5$ where $p^{0}=0$. In the case of $g_{0}=0.1$ we have $p^{0}=5$.

In Fig. $3 \mathrm{~b}$ we draw $q_{1}$ as a function of the deformation parameter $A$ because $q_{1}$ does not depend on $g_{0}$. The minimum value of $q_{1}$ is obtained for $A \approx 1.5$ (positive charge) and $A \approx 4.5$ (negative charge). In the case of $A=-1$ we have $q_{1}=3$ and for $A=-2$ we have $q_{1} \approx 3.5$. For negative $A$ we have positive $q_{1}$ as well as $0 \leq A<3$, while for $A>3$ we have negative $q_{1}$.

Again in Fig. 3c we draw $q_{2}$ in terms of $g_{0}$ and see that $g_{0}<0.3$ yields a negative electric charge, while for the case of $g_{0}>0.3$ we have positive $q_{2}$, which is an increasing function of $g_{0}$. Hence, in the allowed region of $g_{0}$ we may have both positive or negative $q_{2}$.

In Fig. $3 \mathrm{~d}$ we can see that $q_{3}$ is totally positive for positive $g_{0}$ and it is an increasing function of $g_{0}$. In the case of $g_{0}=$ 0.1 we have $q_{3}=6.9$.

In Fig. 4 we draw the entropy (4.1) and see that it is a decreasing function of $g_{0}$ and yields a constant value for large $g_{0}$. For the selected value of the model parameter, we should choose a negative $A$ to have positive entropy. Analyzing the specific heat shows that the black hole is in the stable phase. We investigate the thermodynamics quantities near the horizon, and we need the local temperature of the 

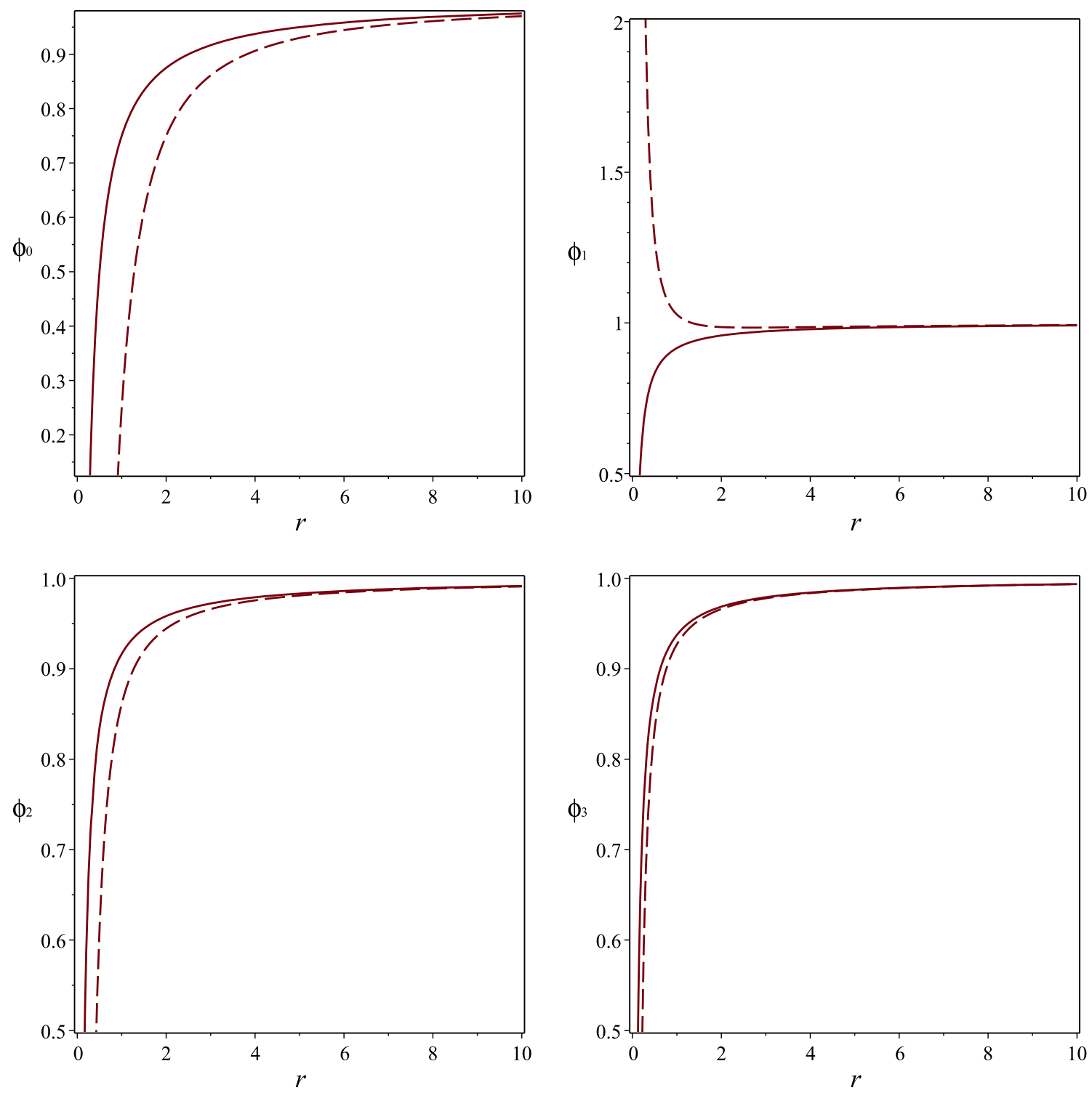

Fig. 2 Typical behavior of scalar fields in terms of $r\left(r \geq r_{+}\right)$for $\omega^{2}=0$. All model parameters are set to unity $\left(a=c=A=g_{0}=g^{1}=\right.$ $\left.g^{2}=g^{3}=\beta^{0}=\beta_{3}=f_{k}=C_{1}=C_{2}=1\right)$. Solid lines denote the first order approximation, while dashed lines represent the second order approximation

black hole, which is drawn in terms of $r$ in Fig. 5. We can see that the black hole temperature is zero at $r=r_{+}=1$, as expected, and it is a decreasing function near the horizon. For the small value of $g_{0}$ (large positive value of magnetic charge), the black hole temperature is a totally decreasing function of $r$. For the larger value of $g_{0}$, the black hole temperature increases first to a maximum value, then decreases to zero at the black hole horizon. The zero magnetic charge case is represented by a solid red line in Fig. 5. In that case, the local temperature is approximately a parabola, hence we can write the local temperature as a function of $r$.

\section{Conductivities}

In this section we try to obtain electrical and thermal conductivities. Obtaining the transport properties depending on the black hole charges (specially magnetic charge) is the main goal of this paper. In order to obtain the electrical conductivity we assume the low frequency limit $\left(\omega^{2} \rightarrow 0\right)$ of the metric (3.1) and use the scalar field (3.18) in Eqs. (2.8) and (2.9). 
(a)

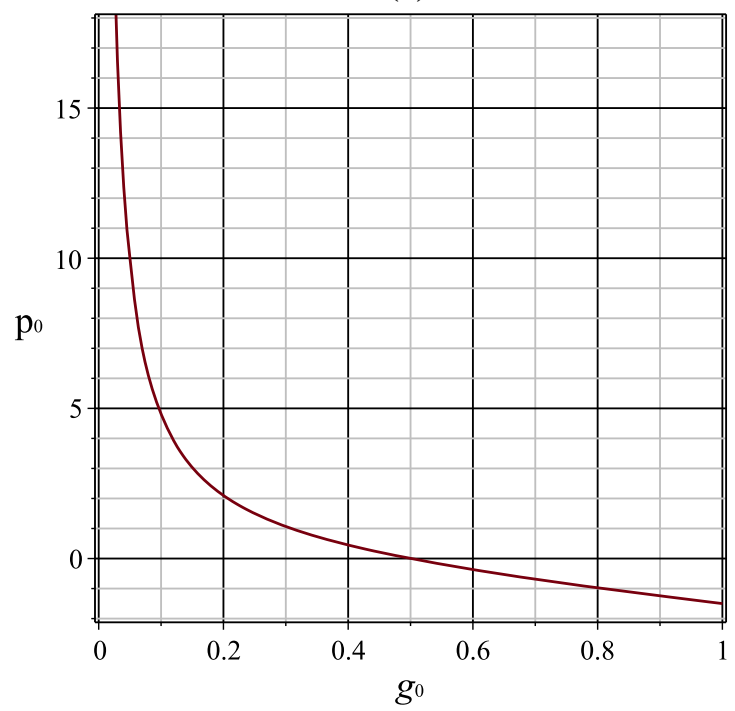

(c)

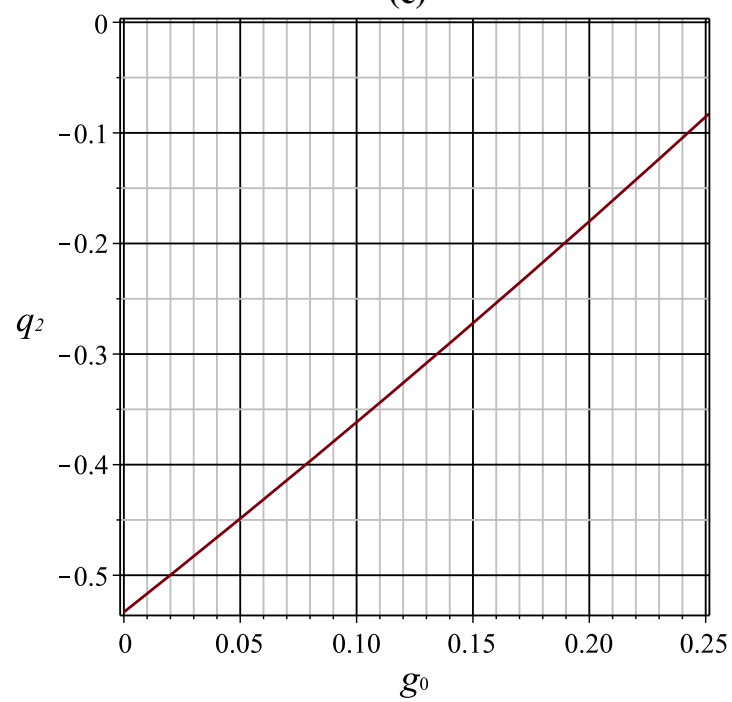

(b)

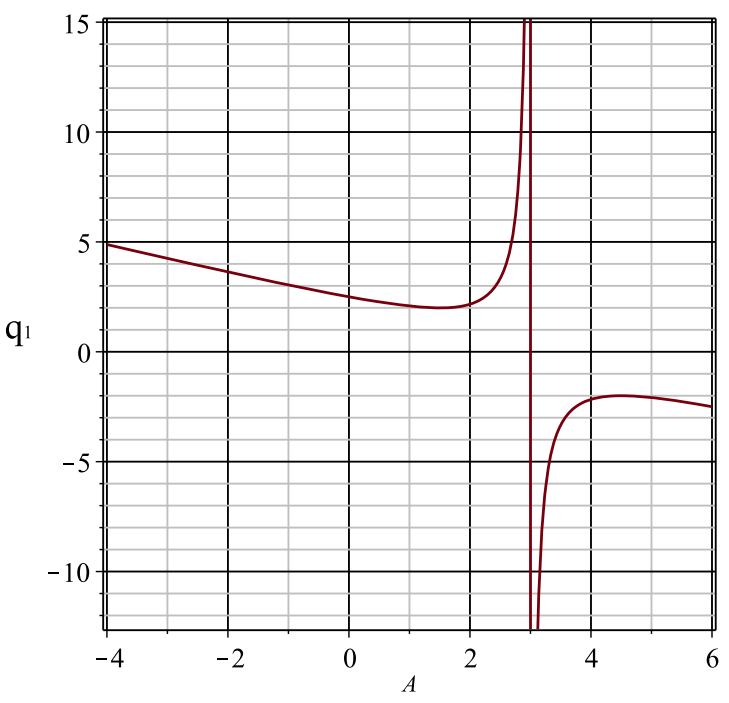

(d)

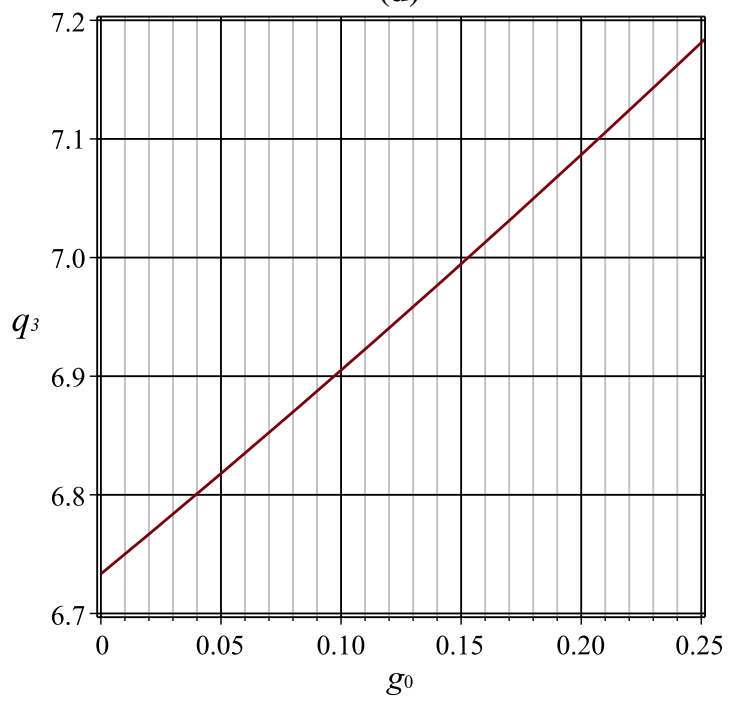

Fig. 3 Magnetic and electric charges in terms of $g_{0}$ for $a=c=g^{1}=g^{2}=g^{3}=\beta^{0}=\beta_{3}=1$ and $A=-2$

\subsection{Electrical conductivity}

Assuming $\left(\phi_{i}\right)^{0}=\left(\phi_{j}\right)^{0}$ gives us similar diagonal and off diagonal conductivity components, hence $\sigma=\sigma_{i i}=\sigma_{i j}$. In the unit of $8 \pi G=1$ and $m=1$ we can obtain the near horizon behavior of the electrical conductivity components, graphically, as illustrated by the plots of Fig. 6. At $T_{C}$, there is a second order phase transition from a normal metal into a superconducting state, which is much like critical phenomena such as superfluids, magnetic ultra- thin films and superconductors.

It is clear that the electrical conductivity is increased below the phase transition temperature, while it is decreased at higher temperature. This means that we have high conductivity at low temperature, as expected.
Also, in Fig. 6a we can find the effect of the magnetic charge on the electrical conductivity near the horizon. In the case of $A=-2$, it has been shown that the maximum conductivity is given by $g_{0} \approx 0.35$, which means $p^{0} \approx 0.5$, $q_{1} \approx 3.5, q_{2} \approx 0.1$ and $q_{3} \approx 7.4$ (see Fig. 3 ).

It means that, in order to have maximum conductivity, the presence of the magnetic charge is necessary. However, decreasing or increasing the magnetic charge decreases the value of electrical conductivity. Thus there is a critical magnetic charge where the maximum of conductivity exists. By using the results of Fig. 5 we can fit the temperature as a function of $r$ and then obtain a plot of the conductivity in terms of the temperature (see Fig. 6b). The asymptotic behavior in Fig. $6 \mathrm{~b}$ shows a superconductor phase transition in the presence of a magnetic charge. 


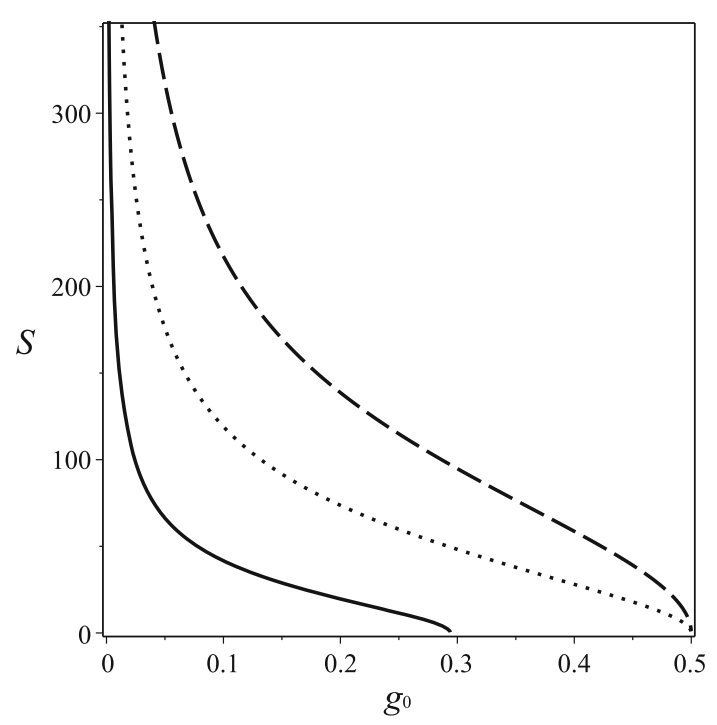

Fig. 4 Typical behavior of entropy in terms of $g_{0}$ for $a=c=g^{1}=$ $g^{2}=g^{3}=\beta^{0}=\beta_{3}=1 . A=-2$ solid, $A=-3$ dotted, $A=-4$ dashed

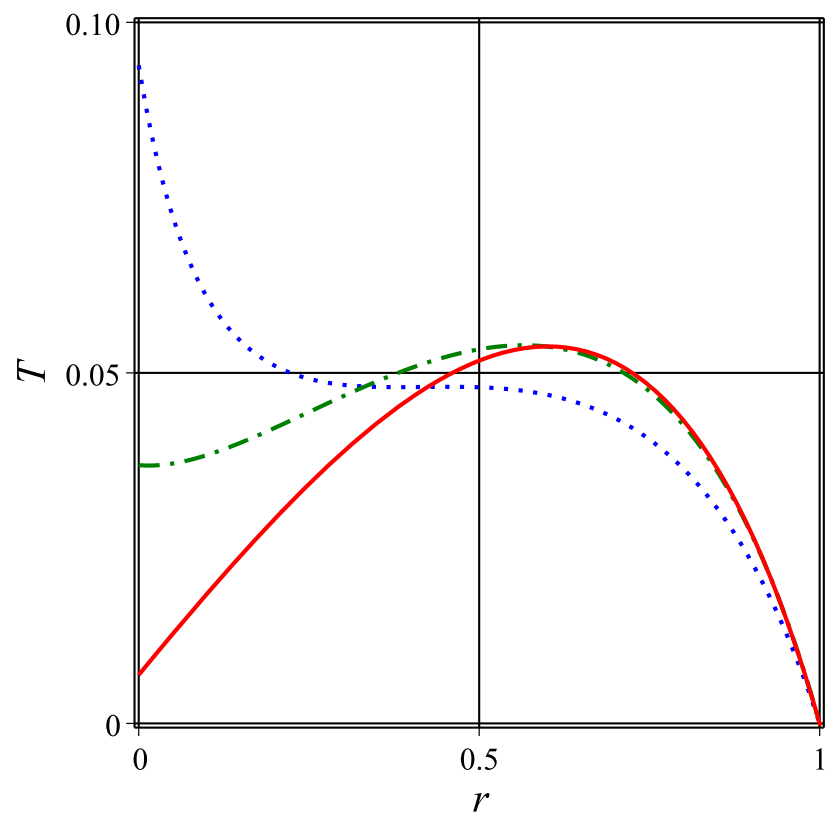

Fig. 5 Typical behavior of local temperature in terms of $r$ for $a=c=$ $g^{1}=g^{2}=g^{3}=\beta^{0}=\beta_{3}=1, A=-2 . g_{0}=0.1$ blue dotted, $g_{0}=0.2$ green dash dotted, $g_{0}=0.5$ (zero magnetic charge) red solid

However, the deformation parameter is also an important quantity in conductivity. We find, for a small negative deformation parameter, that superconductivity occurs for a large positive value of magnetic charge. This means that, for an infinitesimal value of the deformation parameter, superconductivity is due to the large value of the magnetic charge. In other words, superconductivity is enhanced near the horizon due to the magnetic charge for the case of the ordinary STU model $(A=0)$. In this case $(A=0)$, we find special cases of superconductivity (see Fig. 7a) with $g_{0} \approx 0.0036$ where $q_{2}=q_{3} \approx 1\left(q_{1}=2.5\right)$ and $p_{0} \approx 137$. In that case $q_{2}$ and $q_{3}$ are interpreted as electron charges, so the value of the magnetic charge is about 137 electron charge. Comparison of Figs. $6 \mathrm{~b}$ and $7 \mathrm{~b}$, which are the conductivities in terms of the temperature, shows that we can have a superconductive phase transition by using an appropriate choice of $A$ and $g_{0}$. These results are in agreement with other experimental results [47].

In Fig. $7 \mathrm{~b}$ we can see the superconductor at $T=0$ with zero magnetic charge, while in the presence of the magnetic charge we have the superconductive phase transition at finite temperature. In summary, one can conclude that origin of superconductivity may be the magnetic charge.

\subsection{Thermal conductivity}

In order to obtain the thermal conductivity using Eq. (2.10) we use the fact that the chemical potential of the STU black hole is proportional to the temperature $\mu^{i} \propto T$ [48]; also we assume that $i T \approx \omega$, because $T \ll 1$ and $\omega^{2} \ll 1$. In that case we can rewrite the thermal conductivity as follows:

$\kappa_{T}=\frac{s^{2}+\left(\sum_{i=0}^{3} \rho_{i}\right)^{2}}{\sum_{i, j=0}^{3} \rho_{i}\left(G_{i j}(\omega)\right)^{-1} \rho_{j}}$,

where Eq. (2.11) is used. In Fig. 8a we draw the thermal conductivity in terms of $g_{0}$ to see the effect of the magnetic charge. We find that the deformation parameter $A$ should be negative to have real thermal conductivity. We find that the thermal conductivity is proportional to the temperature. It means that decreasing the temperature reduces the value of the thermal conductivity, as expected. The effect of the magnetic charge on the thermal conductivity is strange. In both cases, of large positive and small positive magnetic charge, there is no thermal conductivity. On the other hand, the thermal conductivity increased dramatically for the case of a large negative magnetic charge. However, it is not a physical case, because we found from Eq. (4.3) that $g_{0} \leq 0.5$ (for the unit values of all parameters as selected in the figures); hence in Fig. $8 \mathrm{~b}$ we can see the behavior of the thermal conductivity in the allowed region of $g_{0}$. We can see a maximum of the thermal conductivity for $g_{0} \approx 0.12$, which means $p^{0} \approx 4.8$. It means that a finite value of the magnetic monopole can yield the highest thermal conductivity.

\section{Conclusion}

In this paper, we considered a non-homogeneous deformation of the STU model in four dimensions including three electric and one magnetic charges and used the fluid/gravity correspondence to study holographic superconductors. We 
(a)

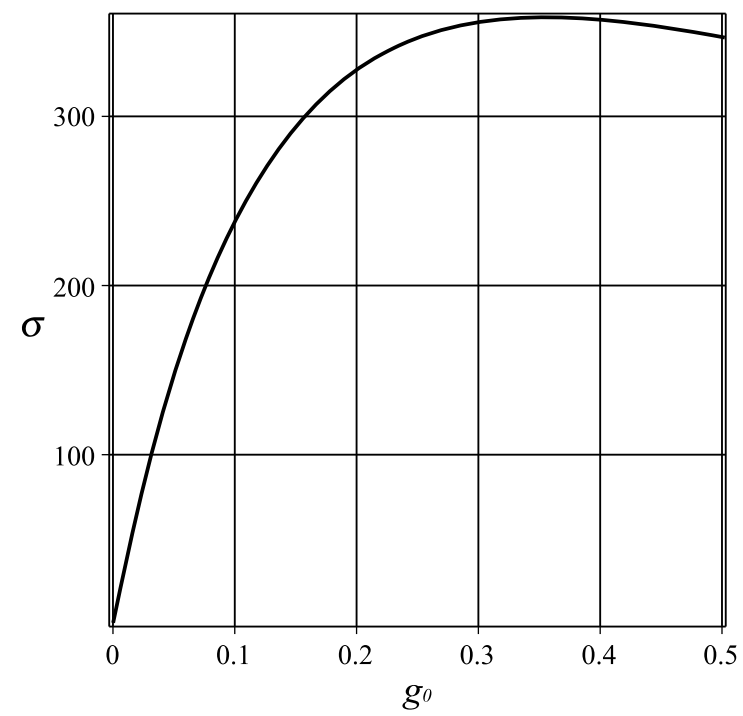

(b)

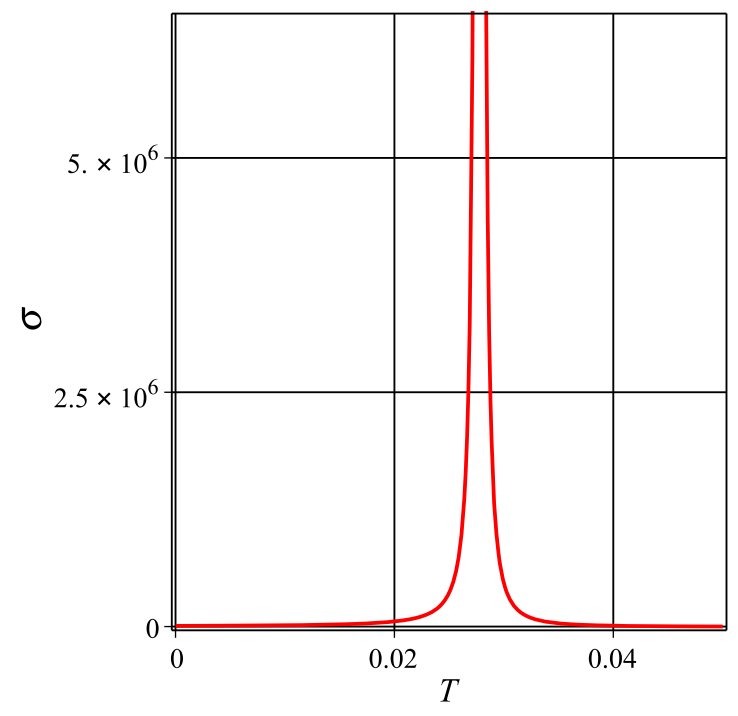

Fig. 6 Typical behavior of electrical conductivity for $\omega^{2}=0, a=c=g^{1}=g^{2}=g^{3}=\beta^{0}=\beta_{3}=f_{k}=1$, and $A=-2$. a In terms of $g_{0}$ with $A=-2$ and $r=1$. $\mathbf{b}$ In terms of $T$ with $A=1.4$ and $g_{0}=0.3$

(a)

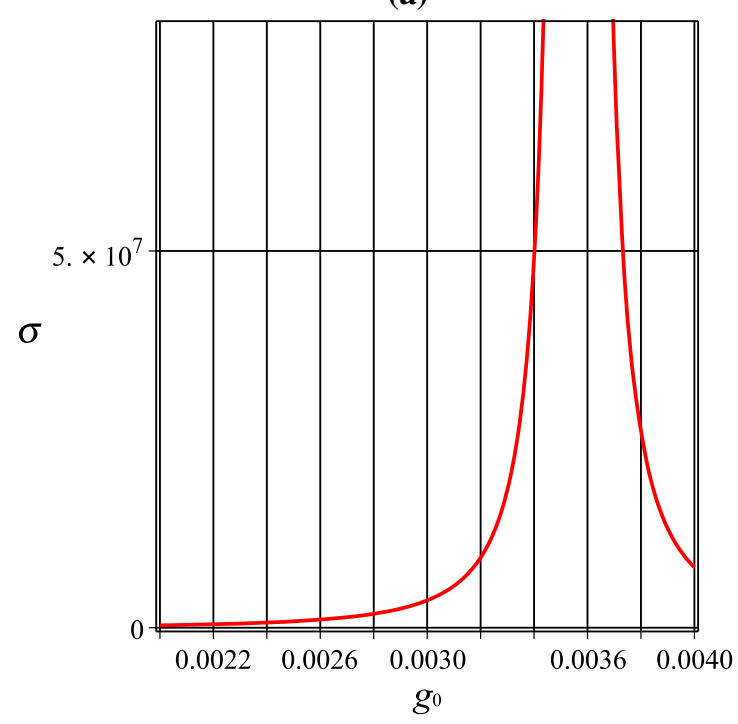

(b)

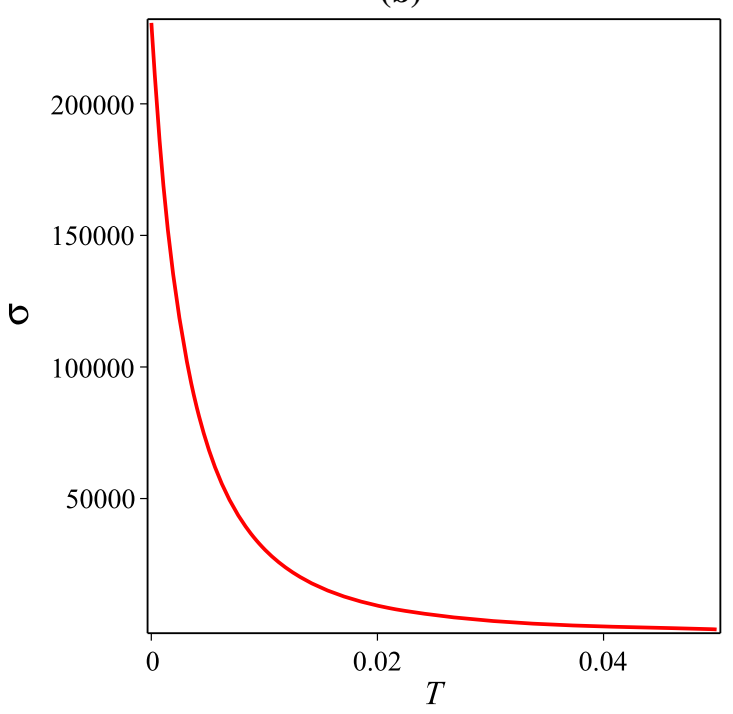

Fig. 7 Typical behavior of electrical conductivity for $\omega^{2}=0, a=c=g^{1}=g^{2}=g^{3}=\beta^{0}=\beta_{3}=f_{k}=1$. a In terms of $g_{0}$ with $A=0$ and $r=1$. b In terms of $T$ with $A=1.4$ and $g_{0}=0.5$ (zero magnetic charge)

obtained transport quantities like the electrical and thermal conductivities and studied the effect of the magnetic charge on them. We focus on the near horizon temperature which is corresponding to low temperature field theory. Numerically, we found the behavior of the scalar field in terms of the radial coordinate, which can be used to interpret the temperature dependent behavior of the scalar field. In that case the near horizon temperature is a decreasing function of the radial coordinate. In order to investigate the effect of the electric and magnetic charges we fixed all parameters and varied only one of the related parameters, which is $g_{0}$. This is one of the order parameters, which correspond to the London penetration depth, while the coherence length is the other order parameter, which corresponds to the radially dependent scalar field. Our numerical analysis has shown that increasing of $g_{0}$ yields a decreasing magnetic charge and vice versa. Hence, we found that the entropy increases by increasing of the magnetic charge. We find that the presence of the magnetic charge is necessary to have high electrical and thermal conductivities, and this may be related to time-dependent perturbations which can enhance superconductivity [49]. A small positive value of the magnetic charge is enough to have 
(a)

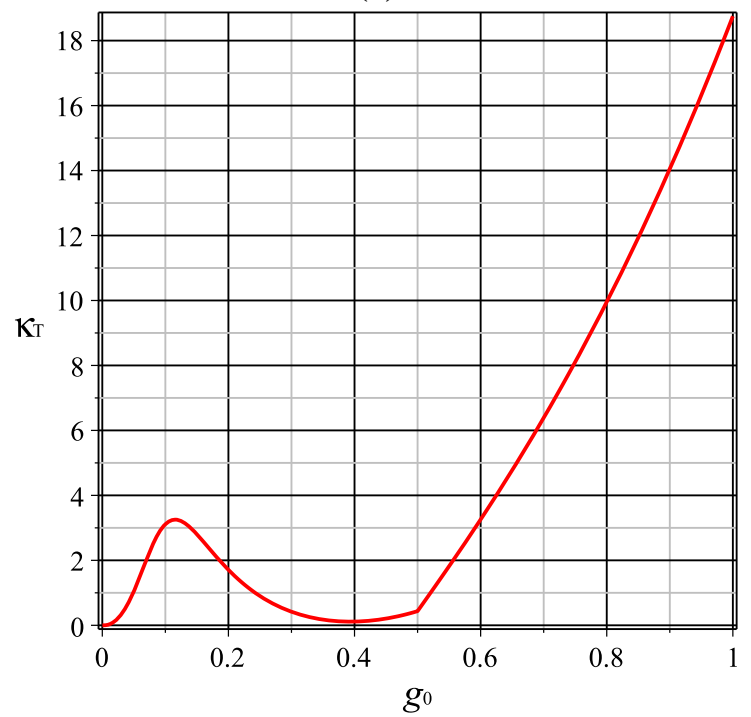

(b)

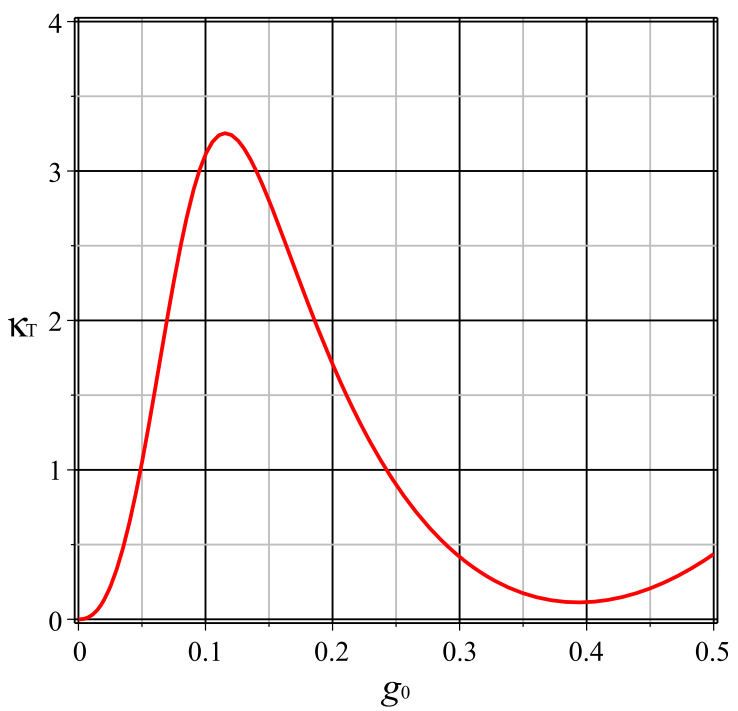

Fig. 8 Thermal conductivity in terms of $g_{0}$ for $\omega^{2}=0, a=c=g^{1}=g^{2}=g^{3}=\beta^{0}=\beta_{3}=f_{k}=1$, $A=-2$, with near horizon behavior $(r \approx 1)$. $\mathbf{a}$ In the extended region, and $\mathbf{b}$ in the allowed region

a maximum of electrical conductivity. Using the numerical analysis, we found the critical value of the magnetic charge where there is a maximum of the electrical conductivity. We have shown that the ordinary STU model $(A=0)$ is better as regards describing a holographic superconductor, however, there are some situations with positive $A$ including a superconductive phase transition.

In order to obtain thermal conductivity we used the ordinary entropy given by (4.1), while it is interesting to calculate thermal conductivity with a logarithmically corrected entropy [50-56] and to study thermal fluctuations corresponding to $N^{-1}$ corrections in AdS/CFT.

Open Access This article is distributed under the terms of the Creative Commons Attribution 4.0 International License (http://creativecomm ons.org/licenses/by/4.0/), which permits unrestricted use, distribution, and reproduction in any medium, provided you give appropriate credit to the original author(s) and the source, provide a link to the Creative Commons license, and indicate if changes were made.

Funded by SCOAP ${ }^{3}$.

\section{References}

1. J.M. Maldacena, The large $\mathrm{N}$ limit of superconformal field theories and supergravity. Int. J. Theor. Phys. 38, 1113 (1999). arXiv:hep-th/9711200

2. E. Witten, Anti-de Sitter space and holography. Adv. Theor. Math. Phys. 2, 253 (1998). arXiv:hep-th/9802150

3. S.S. Gubser, I.R. Klebanov, A.M. Polyakov, Gauge theory correlators from noncritical string theory. Phys. Lett. B 428, 105 (1998). arXiv:hep-th/9802109

4. R.A. Janik, AdS/CFT and the dynamics of quark-gluon plasma. Prog. Theor. Phys. Suppl. 186, 534 (2010). arXiv:1101.0419
5. J. Sadeghi et al., Application of AdS/CFT in quark-gluon plasma. Adv. High Energy Phys. 2013, 759804 (2013)

6. K. Hashimoto, T. Sakai, S. Sugimoto, Nuclear force from string theory. Prog. Theor. Phys. 122, 427 (2009). arXiv:0901.4449

7. S.A. Hartnoll, C.P. Herzog, G.T. Horowitz, Building an AdS/CFT superconductor. Phys. Rev. Lett. 101, 031601 (2008). arXiv:0803.3295

8. S.A. Hartnoll, C.P. Herzog, G.T. Horowitz, Holographic superconductors. JHEP 0812, 015 (2008). arXiv:0810.1563

9. T. Albash, C.V. Johnson, A holographic superconductor in an external magnetic field. JHEP 0809, 121 (2008). arXiv:0804.3466

10. M. Montull, A. Pomarol, P.J. Silva, The holographic superconductor vortex. Phys. Rev. Lett. 103, 091601 (2009). arXiv:0906.2396

11. X.H. Ge, B. Wang, S.F. Wu, G.H. Yang, Analytical study on holographic superconductors in external magnetic field. JHEP 1008, 108 (2010). arXiv: 1002.4901

12. X.H. Ge, H.Q. Leng, Analytical calculation on critical magnetic field in holographic superconductors with backreaction. Prog. Theor. Phys. 128, 1211 (2012). arXiv:1105.4333

13. G.T. Horowitz, Introduction to holographic superconductors. Lect. Notes Phys. 828, 313 (2011). arXiv:1002.1722

14. R.-G. Cai, L. Li, L.-F. Li, R.-Q. Yang, Introduction to holographic superconductor models. Sci. China Phys. Mech. Astron. 58, 060401 (2015). arXiv:1502.00437

15. R.D. Parks, Superconductivity (Marcel Dekker Inc., New York, 1969)

16. H. Rogalla, P.H. Kes, 100 Years of Superconductivity (CRC Press, Taylor \& Francis Group, Boca Raton, 2011)

17. D. Shoenberg, Superconductivity (University Press, Cambridge, 1952)

18. P.B. Price, E.K. Shirk, Evidence for detection of a moving magnetic monopole. Phys. Rev. Lett. 35, 487 (1975)

19. V.R. Malkus, The Interaction of the Dirac Magnetic Monopole with Matter. The Physical Review, Second Series, vol. 83, No. 5 (1951) [for Advanced Studies Series A: Theoretical Physics, 27]

20. B. Cabrera, First results from a superconductive detector for moving magnetic monopoles. Phys. Rev. Lett. 48, 1378 (1982)

21. G. Rickhayzen, Theory of Superconductivity (Wiley, New York, 1964) 
22. L. Hoddeson, E. Braun, J. Teichmann, S. Weart (eds.), An Extensive Overview of the History of Condensed Matter Physics (Oxford University Press, New York, 1992)

23. F. London, Super-Fluids, vol. I (Dover, New York, 1961)

24. O. Domnech et al., Emergent gauge fields in holographic superconductors. JHEP 1008, 033 (2010). arXiv:1005.1776

25. M. Montull et al., Magnetic response in the holographic insulator/superconductor transition. JHEP 1204, 135 (2012). arXiv: 1202.0006

26. A. Salvio, Holographic superfluids and superconductors in dilatongravity. JHEP 1209, 134 (2012). arXiv: 1207.3800

27. A. Salvio, Transitions in dilaton holography with global or local symmetries. JHEP 1303, 136 (2013). arXiv: 1302.4898

28. R.-G. Cai, R.-Q. Yang, Understanding strongly coupling magnetism from holographic duality. Int. J. Mod. Phys. D 25, 1645011 (2016). arXiv: 1601.02936

29. R.-G. Cai, R.-Q. Yang, F.V. Kusmartsev, Holographic model for antiferromagnetic quantum phase transition induced by magnetic field. Phys. Rev. D 92, 086001 (2015). arXiv:1501.04481

30. R.-G. Cai, R.-Q. Yang, A holographic model for paramagnetism/antiferromagnetism phase transition. Phys. Rev. D 91, 086001 (2015). arXiv: 1404.7737

31. D. Klemm, A. Marrani, N. Petri, C. Santoli, BPS black holes in a non-homogeneous deformation of the stu model of $N=2, D=4$ gauged supergravity. JHEP 1509, 205 (2015). arXiv: 1507.05553

32. K. Behrndt, A.H. Chamseddine, W.A. Sabra, BPS black holes in $N=2$ five dimensional AdS supergravity. Phys. Lett. B 442, 97 (1998). arXiv:hep-th/9807187

33. K. Behrndt, M. Cvetic, W.A. Sabra, Non-extreme black holes of five dimensional $N=2$ AdS supergravity. Nucl. Phys. B 553, 317 (1999). arXiv:hep-th/9810227

34. J. Sadeghi, B. Pourhassan, Drag force of moving quark at the $\mathcal{N}=$ 2 supergravity. JHEP 0812, 026 (2008). arXiv:0809.2668

35. J. Sadeghi et al., Drag force of moving quark in STU background. Eur. Phys. J. C 61, 527 (2009). arXiv:0901.0217

36. J. Sadeghi, M.R. Setare, B. Pourhassan, Drag force with different charges in STU background and AdS/CFT. J. Phys. G Nucl. Part. Phys. 36, 115005 (2009). arXiv:0905.1466

37. K. Bitaghsir Fadafan, B. Pourhassan, J. Sadeghi, Calculating the jet-quenching parameter in STU background. Eur. Phys. J. C 71, 1785 (2011). arXiv: 1005.1368

38. B. Pourhassan, J. Sadeghi, STU/QCD correspondence. Can. J. Phys. 91, 995 (2013). arXiv:1205.4254

39. H. Saadat, B. Pourhassan, Holographic superfluid and STU model. Int. J. Theor. Phys. 52, 997 (2013)
40. S. Jain, Holographic electrical and thermal conductivity in strongly coupled gauge theory with multiple chemical potentials. JHEP 1003, 101 (2010). arXiv:0912.2228

41. A. Pourdarvish et al., Statistical analysis of STU black holes. Int. J. Theor. Phys. 53, 1814 (2014)

42. D. Musso, Introductory notes on holographic superconductors. PoS Modave 2013, 004 (2013). arXiv: 1401.1504

43. N. Iqbal, H. Liu, Universality of the hydrodynamic limit in AdS/CFT and the membrane paradigm. Phys. Rev. D 79, 025023 (2009). arXiv:0809.3808

44. R.G. Cai, S. He, L. Li, L.F. Li, A holographic study on vector condensate induced by a magnetic field. JHEP 1312, 036 (2013). arXiv: 1309.2098

45. R.G. Cai, L. Li, L.F. Li, A holographic P-wave superconductor model. JHEP 1401, 032 (2014). arXiv: 1309.4877

46. S.L. Cacciatori, D. Klemm, Supersymmetric $A d S_{4}$ black holes and attractors. JHEP 1001, 085 (2010). arXiv:0911.4926

47. J. Bardeen, L. Cooper, J.R. Schriffer, Theory of superconductivity. Phys. Rev. 8, 1178 (1957)

48. K. Kontoudi, G. Policastro, Diffusion constant of supercharge density in $\mathrm{N}=4 \mathrm{SYM}$ at finite chemical potential. JHEP 1211, 044 (2012). arXiv:1206.2067

49. M. Natsuume, T. Okamura, The enhanced holographic superconductor: is it possible? JHEP 1308, 139 (2013). arXiv:1307.6875

50. A. Pourdarvish et al., Thermodynamics and statistics of Godel black hole with logarithmic correction. Int. J. Theor. Phys. 52, 3560 (2013)

51. J. Sadeghi et al., Logarithmic corrections of charged hairy black holes in $(2+1)$ dimensions. Can. J. Phys. 92, 1638 (2014)

52. B. Pourhassan, M. Faizal, Thermal fluctuations in a charged AdS black hole. Europhys. Lett. 111, 40006 (2015). arXiv:1503.07418

53. M. Faizal, B. Pourhassan, Correction terms for the thermodynamics of a black Saturn. Phys. Lett. B7 51, 487 (2015). arXiv:1505.02373

54. B. Pourhassan, M. Faizal, U. Debnath, Effects of thermal fluctuations on the thermodynamics of modified hayward black hole. Eur. Phys. J. C 76, 145 (2016). arXiv: 1603.01457

55. B. Pourhassan, M. Faizal, Effect of thermal fluctuations on a charged dilatonic black Saturn. Phys. Lett. B 755, 444 (2016). arXiv: 1605.00924

56. J. Sadeghi et al., P-V criticality of logarithm-corrected dyonic charged AdS black holes. Phys. Rev. D 94, 064006 (2016). arXiv: 1605.03458 